\title{
ASO Author Reflections: Predictive Factors for Surgical Treatment Delay in Localised Breast Cancer
}

\author{
María Padilla-Ruiz, $\mathrm{MSE}^{1}$, and Maximino Redondo, $\mathrm{MD}, \mathrm{PhD}^{2}$ \\ ${ }^{1}$ Research Unit, REDISSEC, Costa del Sol Hospital, Marbella, Spain; ${ }^{2}$ Research Unit, REDISSEC, Costa del Sol Hospital, \\ Málaga, Spain
}

\section{PAST}

Treatment delay for patients with breast cancer impacts on health and produces stress among patients; therefore, it is of vital importance to understand the factors involved. Previous studies have found some clinical-pathological factors associated with surgical treatment delay. ${ }^{1,2}$ Unlike previous research in this field, the present study prospectively evaluates a large number of patients diagnosed and treated in different public-sector hospitals. ${ }^{3}$ Sociodemographic parameters, clinical characteristics and lifestyle habits were studied. ${ }^{4}$

\section{PRESENT}

Over half of the study population experienced surgical treatment delay ( $>30$ days). Patients with no formal education, widows, people not requiring assistance for usual activities, those with anxiety or depression, a high BMI, comorbidities, those who were symptomatic, patients with histology different to ductal infiltrating carcinoma or those with poorly differentiated carcinomas were related to a greater surgical treatment delay. ${ }^{4}$

(C) Society of Surgical Oncology 2020

First Received: 9 November 2020

Accepted: 10 November 2020;

Published Online: 24 November 2020

M. Redondo, MD, $\mathrm{PhD}$

e-mail: mredondo@hcs.es

\section{FUTURE}

In view of the findings obtained in this research, the implementation of measures to reduce the surgical waiting times for patients with breast cancer is recommended. Our identification of factors that influence surgical delay highlights the need to raise awareness of their importance among the population at risk and among healthcare professionals. In addition, more attention should be paid to patients with little formal education, in order to enhance the clinical management of their cancer.

DISCLOSURE The authors declare that they have no conflict of interest.

\section{REFERENCES}

1. Bleicher RJ. Timing and delays in breast cancer evaluation and treatment. Ann Surg Oncol. 2018; 25(10):2829-38.

2. Baena-Canada JM, Rodriguez-Perez L, Gamez-Casado S, QuilezCutillas A, Cortes-Carmona C, Rosado-Varela P, et al. Evaluation of waiting times for breast cancer diagnosis and surgical treatment. Clin Transl Oncol. 2018;20(10):1345-52.

3. García-Gutierrez S, Orive M, Sarasqueta C, Legarreta MJ, Gonzalez N, Redondo M, et al. Health services research in patients with breast cancer (CAMISS-prospective): study protocol for an observational prospective study. BMC Cancer. 2018;18(1):54.

4. Padilla-Ruiz M, Zarcos-Pedrinaci I, Rivas-Ruiz F, Téllez-Santana T, García-Gutiérrez S, González N, et al. Factors that influence treatment delay for patients with breast cancer. Ann Surg Oncol. 2020. https://doi.org/10.1245/s10434-020-09409-2.

Publisher's Note Springer Nature remains neutral with regard to jurisdictional claims in published maps and institutional affiliations. 